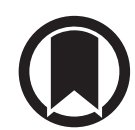

CrossMark

\title{
Transcriptional biomarkers for predicting development of tuberculosis: progress and clinical considerations
}

\author{
Hanif Esmail (10 ${ }^{1,2,3}$, Frank Cobelens ${ }^{4}$ and Delia Goletti ${ }^{5}$ \\ Affiliations: ${ }^{1}$ Medical Research Council Clinical Trials Unit at University College London, London, UK. \\ ${ }^{2}$ Institute for Global Health, University College London, London, UK. ${ }^{3}$ Wellcome Centre for Infectious Diseases \\ Research in Africa, Institute of Infectious Diseases and Molecular Medicine, University of Cape Town, Cape \\ Town, South Africa. ${ }^{4}$ Dept of Global Health and Amsterdam Institute for Global Health and Development, \\ Academic Medical Center, University of Amsterdam, Amsterdam, The Netherlands. ${ }^{5}$ Translational Research \\ Unit, Dept of Epidemiology and Preclinical Research, "L. Spallanzani" National Institute for Infectious \\ Diseases (INMI), IRCCS, Rome, Italy.
}

Correspondence: Delia Goletti, “L. Spallanzani” National Institute for Infectious Diseases, Translational Research Unit, Via Donna Olimpia 195, Rome 00152, Italy. E-mail: delia.golettidinmi.it

@ERSpublications

The new experimental tools to detect incipient tuberculosis (TB) in those with latent TB infection can be clinically relevant for achieving global TB control. In this manuscript, how such tests fit may into future TB management is discussed. http://bit.ly/35WiozD

Cite this article as: Esmail H, Cobelens F, Goletti D. Transcriptional biomarkers for predicting development of tuberculosis: progress and clinical considerations. Eur Respir J 2020; 55: 1901957 [https:// doi.org/10.1183/13993003.01957-2019].

\begin{abstract}
Introduction
Achieving the ambitious targets for global tuberculosis (TB) control, will require an increased emphasis on preventing development of active disease in those with latent TB infection (LTBI) by preventative treatment or vaccination [1]. New shortened regimens of 1-3 months duration potentially allow much wider and more effective use of preventive therapy [2,3]. A significant barrier is the limited ability to reliably identify those at high risk of disease progression, leading to high numbers needed to treat (NNT) to prevent a case of TB [4]. Hence, there is an interest in developing new diagnostic tests that better predict TB disease to allow more targeted preventive treatment and lower NNT [5]. These are often referred to as tests for incipient TB, stemming from the notion that TB prediction with low NNT most likely reflects detection of an early inflammatory response to multiplying Mycobacterium tuberculosis [6]. There are a number of promising biomarkers in development and undergoing evaluation as incipient $\mathrm{TB}$ tests [7]. Although it will be a few years before validated and approved diagnostics are ready for use in clinic, there is the potential for such tests to transform the TB management paradigm and provide a fresh approach to TB control. However, how such tests fit into future TB management and control algorithms is not fully defined and there remain limitations in their potential utility as well as a number of research gaps in this area.
\end{abstract}

\section{How do predictive tests work?}

Infection with $M$. tuberculosis results in an acquired immune response which enables effective granulomatous control of the organism and prevents clinical disease in approximately $90 \%$ of cases. Diagnosis of latent infection relies on detection of this antigen-specific response either in vivo with 
tuberculin skin testing (TST) or ex vivo with interferon- $\gamma$ release assay (IGRA). However, these tests have a poor positive predictive value (on average $2-3 \%$ over 3 years) which worsens with time since initial infection $[8,9]$. Although predictive value is reported to be better in children who are more likely to be tested soon after time of infection, providing treatment to those with evidence of LTBI reduces risk of subsequent disease development but the NNT is high (36-179 depending on the incidence of TB in the setting and test), leading to unnecessary adverse events and high associated cost. Increasing cut-off values of IGRA or calculating monocyte/lymphocyte ratio from routine full blood counts have been demonstrated to identify those at increased risk of disease development, but these approaches unsurprisingly have poor sensitivity. A recent, World Health Organization (WHO)-approved target product profile (TPP) recommended that for novel tests to predict TB development, both sensitivity and specificity for active disease progression over a 2 year period should be at least $75 \%$ (minimally) to $90 \%$ (optimally), which would reduce the NNT to approximately 10-25 [10, 11].

To understand how these novel predictive tests might work requires consideration of TB disease evolution. Granuloma may succeed in sterilising infection but in many of those initially infected, viable bacilli are thought to persist (although it is notable that we have no test to determine presence of viable bacilli and the proportion in whom this persists is therefore unknown). If granulomatous control fails, the subsequent increasing bacillary load and the host cellular infiltration in response to this drive development of local pathology and systemic illness. However, data show that the period between initiation of this disease process and development of symptoms and presentation to the health service can take months if not years. TB prevalence surveys highlight that most of those found in the community with culture-positive disease are not seeking healthcare and deny typical TB symptoms but have visible abnormalities on chest radiograph (CXR) [12]. Studies utilising positron-emission tomography or computed tomography (PET/ CT) imaging have shown parenchymal lesions suggestive of disease in those that subsequently develop disease prior to visible CXR lesions [13]. There is therefore a window for early detection of disease, where the individual is well, unaware of symptoms and not seeking healthcare, akin to screening approaches for cancer. Detecting these early stages of disease to identify those at risk of future clinical presentation is the most feasible approach at present.

\section{Transcriptional biomarkers for predicting disease development}

A number of approaches have been utilised for diagnostic development which include 1) detecting low levels of $M$. tuberculosis antigens or nucleic acid, 2) identifying $M$. tuberculosis-specific immunophenotypes that correlate with disease risk and 3) detecting host RNA or protein signatures sensitive for early disease [14-16]. In contrast to the T-cell-based re-stimulation approach used in IGRA, a more global consideration of the host response in unstimulated blood using transcriptomics to evaluate mRNA abundance, have proved most successful for developing biomarkers of early disease. Over the past decade a large number of studies utilising RNA sequencing or microarray for biomarker discovery have identified mRNA transcript signatures in whole blood that distinguish those with clinical, active pulmonary TB from those with latent infection or other diseases [17]. These approaches characterise the differences in the global host response between disease states, reflecting both the changes in gene expression in circulating immune cells and their relative abundance in the blood sample. Of note, transcriptional changes have been shown to normalise with treatment of active TB [18]. In addition, and importantly for predicting disease, for a subset of the active TB-associated transcripts, changes in abundance were detectable in asymptomatic individuals in whom early TB pathology was demonstrated using PET/CT imaging, prior to clinical disease development [19]. Cohort studies following at-risk populations have also shown that transcriptional signatures can distinguish progressors from non-progressors from at least a year before clinical presentation [20]. While exact transcript signatures defined by these studies has differed with different study populations, aims and bioinformatic approaches, there is considerable overlap and co-correlation of transcripts, and the biological processes reflected by them is consistent $[21,22]$.

While in initial studies several signatures published met the WHO thresholds [7], they appear to perform less well in study populations outside those in which they were discovered. A recent meta-analysis evaluating 17 published candidate TB signatures against a pooled dataset from four studies containing 127 cases of incipient TB, identified eight signatures that performed equivalently. However, all failed to meet the minimal standard of $75 \%$ sensitivity and specificity for disease progression at 2 years proposed in the WHO TPP [22].

These limitations in part reflect two important features of disease progression, namely a significant proportion of individuals who develop incipient disease will self-heal and not progress registering a false-positive test. Secondly, progression rates of disease are heterogenous. While in some progression may take years, those that develop disease more rapidly will only test positive close to onset of clinical disease. 
Indeed, the positive predictive value of the eight identified signatures for disease development was 11.1-14.3\% over 3 months, 9.5-11.7\% over 6 months and $6.8-9.4 \%$ over 24 months [22]. Assuming $60 \%$ treatment efficacy $[23,24]$ these values translate to NNTs of $12-15,14-18$ and $18-25$, respectively, but it should be kept in mind that they are population-dependent (mainly TB incidence-dependent, if not considering host immune suppression) (figure 1).

In addition, there are inherent issues with the sensitivity and specificity of the transcriptomic approach. Gene expression is determined by the nature of the host response; however, not all forms of TB share a common disease pathogenesis. As a result, the transcriptional response may differ in miliary disease, in disease presentations in early childhood or in immunocompromised hosts. It is notable that sensitivity of these transcriptional signatures is $\sim 90 \%$ even in confirmed active disease, which inherently limits the sensitivity for incipient disease [25]. Furthermore, other diseases share elements of the whole blood transcriptional responses found in $\mathrm{TB}$, limiting specificity of the tests. In particular, the interferon signalling, which is the most enriched pathway in many of the TB transcriptional signatures, is also an important component of the transcriptional response in untreated HIV infection [19].

Finally, for these biomarker signatures to have a role in clinical care, several steps are needed to translate these into assays for routine use [26]. Whole genome discovery approaches need to be translated to more tractable, such as multiplex PCR or NanoString's Counter technology, for the most parsimonious sets of predictive transcripts. Scoring methods must be determined to provide diagnostic cut-off values and labour-intensive RNA extraction methods need to be streamlined and ideally integrated into the diagnostic platform. Progress is being made, with companies such as Cepheid developing cartridges to monitor host mRNA abundance by real time PCR on the GeneXpert platform. These assays, once developed, should then be evaluated in prospective studies in populations of their intended use for their clinical and public health utility [10] However, it is also important to reflect that the challenges in overcoming all these steps means that numerous promising biomarkers fail to progress to the clinic. Transcriptional biomarkers are slowly making their way into clinical practice primarily in the cancer field where a number of commercial products are available to measure transcript abundance, mainly in tissue samples. Additionally, although at this stage transcriptional biomarkers for the purpose of short term prediction of disease progression look most promising, it may be that other approaches, particularly T-cell activation markers or biomarkers

Latent TB test

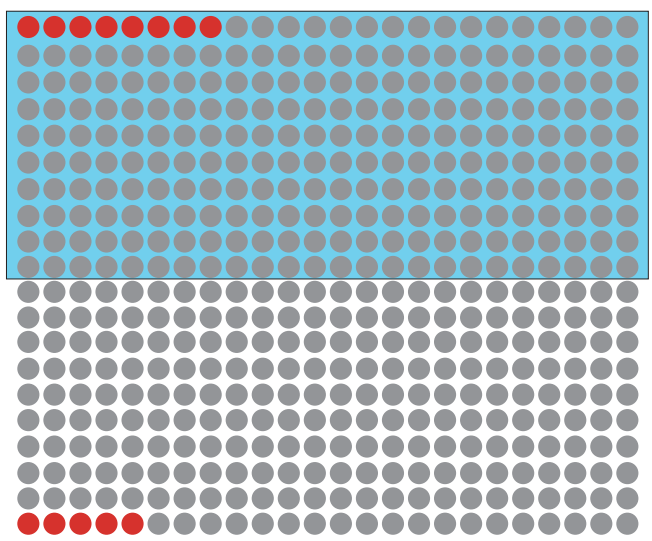

Incipient TB test

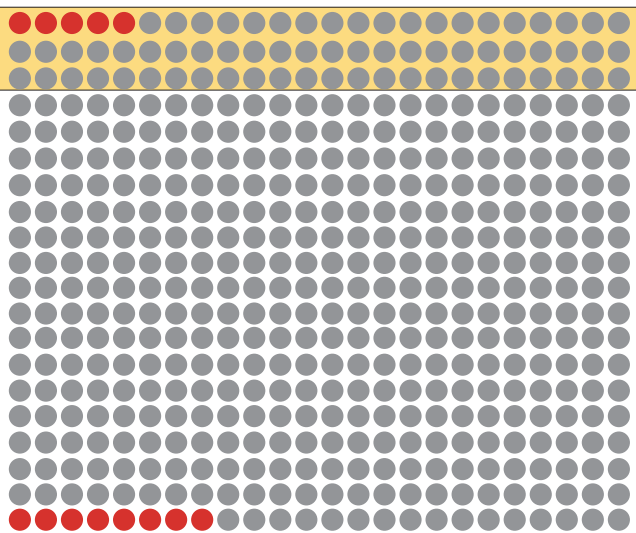

No disease at 24 months

- Incident disease over 24 months

Baseline test for latent TB - proportion positive

Baseline test for incipient TB - proportion positive

FIGURE 1 In this illustrative scenario, 480 individuals are tested for latent tuberculosis (TB) and incipient TB at baseline. Among them, 13 (2.7\%) develop incident TB over 24 months. At baseline $50 \%$ have a positive test for latent TB whereas 15\% have a positive test for incipient TB. At 24 months, the latent TB test accuracy for predicting incident cases has a sensitivity of $62 \%$, specificity $50 \%$, positive predictive value (PPV) $3.3 \%$ and negative predictive value (NPV) $98 \%$. In contrast, the incipient TB test for predicting incident cases has a sensitivity $39 \%$, specificity $86 \%$, PPV $6.9 \%$ and NPV $98 \%$. The number needed to treat to prevent disease within 24 months respectively would be 50 for the latent TB test (with five missed cases) and 24 for the incipient TB test (with eight missed cases). Note this does not take into account reinfection over the 24-month period. 
derived from proteomic studies or other approaches that are in early development, may be more fruitful in the longer term.

\section{Potential clinical use of tests}

Whole blood transcriptional biomarkers mark clear progress in predicting clinical progression of disease. If the minimal test performance recommended by WHO can be attained and technical challenges overcome, transcriptional biomarker-based tests hold promise for selecting high TB risk individuals for preventive therapy. In figure 1 we show a potential use of these assays. In low-incidence settings these could include household, school and occupational contacts of infectious TB patients, healthcare workers and recent immigrants from high-incidence countries. In high-incidence settings, incipient TB tests could additionally be used for community screening [27], for example in (bi-)annual campaigns. Because of a time-lag effect in which an inflammatory response may commence only months after $M$. tuberculosis infection, repeat testing may be warranted. As a result, we may need to reconsider our approach to evaluating and testing risk groups such as household contacts, who are typically only assessed for evidence of TB infection (with IGRA or TST) and consideration for preventive therapy at baseline. A new algorithm, incorporating novel predictive diagnostics testing, may require repeated testing at 6 monthly or yearly intervals for a period of 2-3 years. Comparison of diagnostic and treatment algorithms should be evaluated in clinical trials, but patient and provider views on the different approaches will also need to be explored. Additionally, technical feasibility and affordability will be important considerations, and cost-effectiveness analyses will be needed to inform policy decisions with regard to target groups and frequency of testing. Transcriptional biomarker-based tests can likely also be used for triaging symptomatic patients or those with abnormal CXR being evaluated for TB, by more precisely identifying those who need further diagnostics for bacteriological confirmation [28], this also being important for cost considerations. As there is no time-lag effect, sensitivity for this use case is expected to be higher than for prediction, whereas specificity may be lower because alternative causes of disease may elicit a similar transcriptional response.

It is as yet unclear whether transcriptional biomarker-based tests should be used to guide preventive therapy in individuals with impaired cell-mediated immunity, such as people living with HIV, pre-transplant patients and those using tumour necrosis factor- $\alpha$ blocking agents [28]. In these target groups contained $M$. tuberculosis infection may quickly progress to severe disease, with a narrow time window to test for incipient TB. Therefore, current approaches to risk stratification, combining epidemiological factors with or without IGRA, may remain appropriate.

Another question that these new assays raise is how incipient TB, defined by a positive prediction test but negative molecular assay or $M$. tuberculosis culture, should be treated. Clinical trials will be required to establish non-inferiority of preventive treatment to full-course chemotherapy, with a potential role for shortened (e.g. 2 months) four-drug regimens [29].

Acknowledgements: This project is part of the Global TB Network activities carried out for the latent tuberculosis group. Moreover, this work is a part of the research performed thanks to the grant of Ricerca Corrente from the Italian Ministry of Health Linea 4, program 2.

Support statement: This work was supported by Ricerca Corrente, Italian Ministry of Health. Funding information for this article has been deposited with the Crossref Funder Registry.

Conflict of interest: H. Esmail has nothing to disclose. F. Cobelens has nothing to disclose. D. Goletti reports grants and personal fees from Quidel, personal fees from Qiagen, Janssen and Diasorin, outside the submitted work.

\section{References}

1 Houben RMGJ, Menzies NA, Sumner T, et al. Feasibility of achieving the 2025 WHO global tuberculosis targets in South Africa, China, and India: a combined analysis of 11 mathematical models. Lancet Glob Health 2016; 4: e806-e815.

2 Martinson NA, Barnes GL, Moulton LH, et al. New regimens to prevent tuberculosis in adults with HIV infection. N Engl J Med 2011; 365: 11-20.

3 Swindells S, Ramchandani R, Gupta A, et al. One month of rifapentine plus isoniazid to prevent HIV-related tuberculosis. N Engl J Med 2019; 380: 1001-1011.

4 Rangaka MX, Wilkinson KA, Glynn JR, et al. Predictive value of interferon- $\gamma$ release assays for incident active tuberculosis: a systematic review and meta-analysis. Lancet Infect Dis 2012; 12: 45-55.

5 Schumacher SG, Denkinger CM. Diagnostic test for incipient tuberculosis: a step forward, many more to go. Am J Respir Crit Care Med 2018; 197: 1106-1107.

6 Cobelens F, Kik S, Esmail H, et al. From latent to patent: rethinking prediction of tuberculosis. Lancet Respir Med 2017; 5: 243-244.

7 Warsinske H, Vashisht R, Khatri P. Host-response-based gene signatures for tuberculosis diagnosis: A systematic comparison of 16 signatures. PLoS Med 2019; 16: e1002786.

8 Diel R, Loddenkemper R, Nienhaus A. Predictive value of interferon- $\gamma$ release assays and tuberculin skin testing for progression from latent TB infection to disease state: a meta-analysis. Chest 2012; 142: 63-75. 
9 Adetifa IM, Ota MOC, Jeffries DJ, et al. Interferon- $\gamma$ ELISPOT as a biomarker of treatment efficacy in latent tuberculosis infection: a clinical trial. Am J Respir Crit Care Med 2013; 187: 439-445.

10 World Health Organization. Consensus Meeting Report: Development of a Target Product Profile (TPP) and a Framework for Evaluation for a Test for Predicting Progression from Tuberculosis Infection to Active Disease. Geneva, World Health Organization, 2017. Available from: www.who.int/tb/publications/2017/tpp_infection_ disease/en/

11 Kik SV, Schumacher S, Cirillo DM, et al. An evaluation framework for new tests that predict progression from tuberculosis infection to clinical disease. Eur Respir J 2018; 52: 1800946. .

12 Onozaki I, Law I, Sismanidis C, et al. National tuberculosis prevalence surveys in Asia, 1990-2012: an overview of results and lessons learned. Trop. Med Int Health 2015; 20: 1128-1145.

13 Esmail H, Lai RP, Lesosky $\mathrm{M}$, et al. Characterization of progressive HIV-associated tuberculosis using 2-deoxy-2-[(18)F]fluoro-D-glucose positron emission and computed tomography. Nat Med 2016; 22: 1090-1093.

14 Goletti D, Lee M-R, Wang J-Y, et al. Update on tuberculosis biomarkers: from correlates of risk, to correlates of active disease and of cure from disease. Respirology 2018; 23: 455-466.

15 Goletti D, Lindestam Arlehamn CS, Scriba TJ, et al. Can we predict tuberculosis cure? What tools are available? Eur Respir J 2018; 52: 1801089.

16 Petruccioli E, Scriba TJ, Petrone L, et al. Correlates of tuberculosis risk: predictive biomarkers for progression to active tuberculosis. Eur Respir J 2016; 48: 1751-1763.

17 Walzl G, McNerney R, du Plessis N, et al. Tuberculosis: advances and challenges in development of new diagnostics and biomarkers. Lancet Infect Dis 2018; 18: e199-e210.

18 Thompson EG, Du Y, Malherbe ST, et al. Host blood RNA signatures predict the outcome of tuberculosis treatment. Tuberculosis (Edinb) 2017; 107: 48-58.

19 Esmail H, Lai RP, Lesosky $\mathrm{M}$, et al. Complement pathway gene activation and rising circulating immune complexes characterize early disease in HIV-associated tuberculosis. Proc Natl Acad Sci USA 2018; 115: E964-E973.

20 Zak DE, Penn-Nicholson A, Scriba TJ, et al. A blood RNA signature for tuberculosis disease risk: a prospective cohort study. Lancet 2016; 387: 2312-2322.

21 Blankley S, Graham CM, Levin J, et al. A 380-gene meta-signature of active tuberculosis compared with healthy controls. Eur Respir J 2016; 47: 1873-1876.

22 Gupta RK, Turner CT, Venturini C, et al. Concise whole blood transcriptional signatures for incipient tuberculosis: a systematic review and patient-level pooled meta-analysis. Lancet Resp Med 2020; in press [https:// doi.org/10.1016/S2213-2600(19)30282-6].

23 Wilkinson D. Drugs for preventing tuberculosis in HIV infected persons. Cochrane Database Syst Rev 2000; CD000171.

24 Stagg HR, Zenner D, Harris RJ, et al. Treatment of latent tuberculosis infection: a network meta-analysis. Ann Intern Med 2014; 161: 419-428.

25 Sweeney TE, Braviak L, Tato CM, et al. Genome-wide expression for diagnosis of pulmonary tuberculosis: a multicohort analysis. Lancet Respir Med 2016; 4: 213-224.

26 Gliddon HD, Herberg JA, Levin M, et al. Genome-wide host RNA signatures of infectious diseases: discovery and clinical translation. Immunology 2018; 153: 171-178.

27 Sumner T, Scriba TJ, Penn-Nicholson A, et al. Potential population level impact on tuberculosis incidence of using an mRNA expression signature correlate-of-risk test to target tuberculosis preventive therapy. Sci Rep 2019; 9: 11126 .

28 Getahun H, Matteelli A, Abubakar I, et al. Management of latent Mycobacterium tuberculosis infection: WHO guidelines for low tuberculosis burden countries. Eur Respir J 2015; 46: 1563-1576.

29 Hong Kong Chest Service/Tuberculosis Research Centre. A controlled trial of 2-month, 3-month, and 12-month regimens of chemotherapy for sputum-smear-negative pulmonary tuberculosis: results at 60 months. Am Rev Respir Dis 1984; 130: 23-28 